\title{
Cues to the usefulness of grooming behavior in the evaluation of anxiety in the elevated plus-maze
}

\author{
Celio Estanislau \\ Universidade Estadual de Londrina, Londrina, PR, Brazil
}

\begin{abstract}
Grooming behavior has been reported to be a response to novelty and other stressors. However, studies that sought to identify anxiety-related measures in the elevated plus-maze have not found grooming as a reliable index. Grooming has been implied with dearousal after a stressful experience. In the present work, a detailed investigation of this behavior was performed in a test with a longer duration (i.e., $10 \mathrm{~min}$ ) than the usual duration in the elevated plus-maze. The level of anxiety was manipulated prior to the test by confining male rats to environments known to differ in the degree of aversive stimulation, including a familiar cage, a closed arm of the elevated plus-maze, and an open arm (i.e., the most aversive arm) of the elevated plus-maze. Grooming behavior was classified into three constituent elements: rostral grooming, head grooming, and body grooming. The groups were compared with regard to conventional measures of the elevated plus-maze and grooming behavior. Prior confinement to an open arm led to a delayed effect of decreased open-arm exploration undetectable during the first half of the test compared with rats previously confined to a closed arm. Prior confinement to an open arm also increased the total duration of grooming during the second half of the test. These increases were found to be partially attributable to increases in the rostral element of grooming. The percentage of interrupted bouts was also found to discriminate the group previously confined to an open arm. These results indicate that prolongation of the session can reveal effects that are otherwise undetectable and that under such conditions some grooming measures can be useful in the evaluation of anxiety-like behavior. Keywords: elevated plus-maze; dearousal, anxiety; grooming behavior
\end{abstract}

Received 2 May 2012; received in revised form 6 June 2012; accepted 8 June 2012. Available online 29 June 2012.

\section{Introduction}

The elevated plus-maze is a widely used behavioral test for the study of anxiety in laboratory rodents (Pellow, Chopin, File, \& Briley, 1985). Its vast use can be credited to its reliable sensitivity to both anxiolytic and anxiogenic treatments, simplicity of the procedure, and its basis on natural tendencies and spontaneous behavior that do not require lengthy training or the use of noxious stimuli or food/water deprivation (Carobrez \& Bertoglio, 2005; Walf \& Frye, 2007). Despite its use in a great number of studies that have contributed to the understanding of anxiety in the past decades, the elevated plus-maze is also a target for criticism because it does not produce consistent results with "novel compounds" (i.e., those not related to $\gamma$-aminobutyric acid [GABA]/benzodiazepine or glutamate receptors; e.g., Borsini, Podhorna, \& Marazziti, 2002). Furthermore, even anxiolytic compounds that regularly produce consistent effects may exhibit a lack of effect

Correspondence regarding this article should be directed to: C. Estanislau, Departamento de Psicologia Geral e Análise do Comportamento, Centro de Ciências Biológicas, Universidade Estadual de Londrina, PR445, Km 380, 86051-990, Londrina, PR, Brazil. Phone: +55 4333714261.Fax: +554333714227; E-mail: estanislau@uel.br in a second elevated plus-maze session, a phenomenon known as "one-trial tolerance" (for review, see Carobrez \& Bertoglio, 2005). These limitations call for the development of new approaches to the study of anxiety in rodents.

Grooming behavior has the essential function of caring for and protecting the surface of the body, but its occurrence can frequently be better explained by considering the context rather than the state of the skin or fur. Indeed, this behavior can be induced by exposure to many stressors or central administration of adrenocorticotropic hormone (for review, see Spruijt, van Hooff, \& Gispen, 1992), highlighting its association with states of stress. Closely related to what occurs with the stress response (Koolhaas, de Boer, Coppens, \& Buwalda, 2010), individual factors also contribute to grooming responsiveness. For example, rats selectively bred for anxious coping in a two-way active avoidance task show increased grooming activity when exposed to novelty compared with rats selected for the opposite trait (Escorihuela, Fernández-Teruel, Gil, Aguilar, Tobeña, \& Driscoll, 1999). Similarly, in an elevated plus-maze session, greater grooming activity is found in rats that spontaneously show low open-arm exploration (Estanislau, Ramos, Ferraresi, Costa, de Carvalho, \& Batistela, 2011). Nonetheless, although many studies 
have evaluated behavioral measures relevant for anxiety in the elevated plus-maze, grooming measures are generally not found among anxiety-related measures (e.g., Cruz, Frei, \& Graeff, 1994; Rodgers \& Johnson, 1995; Setem, Pinheiro, Motta, Morato, \& Cruz, 1999).

In recent years, an in-depth approach to grooming behavior has been proposed as useful in the behavioral investigation of stress and anxiety (Kalueff \& Tuohimaa, 2004, 2005a). Beyond the evaluation of the latency, frequency, and duration of grooming, it also consists of studying other characteristics such as the occurrence of short interruptions) and individually investigating and sequencing its different constituent elements. This approach can be fruitful. For example, some of these measures are bidirectionally sensitive to GABAergic drugs (Kalueff \& Tuohimaa, 2005b). However, they also produced inconsistent effects with acute antidepressant treatments (e.g., amitriptyline and fluoxetine; Enginar, Hatipoğlu, \& Firtina, 2008), suggesting that the grooming/ anxiety relationship deserves further evaluation including nonpharmacological studies.

The objective of the present study was to investigate the grooming response as an index of anxiety in the elevated plus-maze. Grooming has been suggested to be involved in dearousal from stressful situations (for review, see Spruijt et al., 1992), and a 5-min session appears to be too short to allow the appropriate examination of this behavior. Therefore, a longer session of 10 min was used in the present work. The anxiety state was manipulated by confining rats to different aversive contexts prior to the session, including an open arm of the elevated plus-maze, a closed-arm of the elevated plus-maze, and a familiar cage. Confinement to an open or closed arm is a novel experience, but the former is known to induce greater increases in plasma corticosterone and anxiety-related behavior (Pellow et al., 1985). It also induces c-fos expression in anxiety-related neural sites (Nguyen, Sartori, Herzog, Tasan, Sperk, \& Singewald, 2009). One may state that confinement to an arm may itself constitute a maze experience that could entirely account for any behavioral alterations found, but a previous study showed that only the experience of being exposed to the entire maze (or at least both one open and one closed arm) is able to increase open-arm avoidance in a subsequent session (Bertoglio \& Carobrez, 2000). The results of this research may be useful to define how grooming behavior can be approached for the study of anxiety.

\section{Methods}

\section{Subjects}

Thirty-four Wistar-derived male rats (250-300 g) were obtained from the Biotério Central of the Universidade Estadual de Londrina. Throughout the experiment, rats were housed six per cage and kept under controlled room temperature $\left(24 \pm 1^{\circ} \mathrm{C}\right)$ and a $12 \mathrm{~h} / 12 \mathrm{~h}$ light/dark cycle (lights on at 7:00 AM). Tap water and commercial rat chow were supplied ad libitum. No procedure was performed before an adaptation period (never shorter than $72 \mathrm{~h}$ ) to our vivarium. Experimental tests were always conducted between 2:00 PM and 6:00 PM. All procedures were performed in accordance with the recommendations of the Brazilian Society of Neuroscience and Behavior that are similar to those of the National Institutes of Health. Additionally, the experiments were approved by the State University of Londrina Ethics Committee for Animal Research (CEEA 07/08).

\section{Apparatus}

Polypropylene cages $(28 \mathrm{~cm} \times 17 \mathrm{~cm} \times 13 \mathrm{~cm})$ were used for the familiarization procedure (see below). Testing was conducted with a wooden elevated plusmaze. Briefly, it consisted of two open arms $(50 \mathrm{~cm} \times 12$ $\mathrm{cm})$ at right angles with two closed arms $(50 \mathrm{~cm} \times$ $12 \mathrm{~cm}$ ) surrounded by $40-\mathrm{cm}$ high wooden walls. A central square $(12 \mathrm{~cm} \times 12 \mathrm{~cm})$ linked the four arms. To avoid falls, the open arms were surrounded by a $1-\mathrm{cm}$ high plexiglas rim. The experimental room was lit by a $60-\mathrm{W}$ bulb placed $2.4 \mathrm{~m}$ above the central square of the maze. The apparatus was cleaned with a $5 \%$ ethanol solution and dried with paper towels between sessions. The elevated plus-maze sessions were video-recorded by a camera placed $2.4 \mathrm{~m}$ above the apparatus.

\section{Procedure}

Familiarization procedure and confinement. All rats were individually kept for $5 \mathrm{~min}$ in small cages daily for 3 days. On the fourth day they were randomly distributed for 5-min confinement under one of three conditions: (1) the same cage as the previous days, (2) a closed arm of the elevated plus-maze, or (3) an open arm of the elevated plus-maze. Confinement to the open or closed arm was achieved by affixing a wooden plate between the arm and the rest of the maze. After the 5 -min confinement, each rat was placed for $60 \mathrm{~s}$ in a small cage that was similar to the one used earlier and then tested for $10 \mathrm{~min}$ in the elevated plus-maze.

In an attempt to prevent, as much as possible, habituation to the maze that results from exposure to a closed or open arm, the maze was rotated $90^{\circ}$ between the confinement and testing of the rats that were confined to these spaces.

Elevated plus-maze. One minute after the 5-min confinement, each rat was tested in a wooden elevated plus-maze. The groups were compared with regard to spatiotemporal measures that were recorded during the session. Thus, the number of entries into (i.e., with all four paws) and time spent on the arms, entries into and time spent in the arm extremities (i.e., distal $20 \mathrm{~cm}$ ), distance traveled on the different types of arms, and time spent on the center square were scored. Because elevated plus-maze sessions are frequently $5 \mathrm{~min}$ long, to allow comparisons with previous studies and explore time-related behavioral changes, the 10-min session was divided into two 5-min blocks. 
Grooming recording. Grooming behavior was classified into three types of constituent elements that constitute a simplified version of the classification used in previous work (Berridge, Fentress, \& Parr, 1987; Berridge, Aldridge, Houchard, \& Zhuang, 2005): rostral grooming (licking of forepaws or their friction with the mouth or nose), head grooming (friction of the forepaws with the ear region), and body grooming (oral friction directed to the trunk, hindpaws, anogenital region, or tail). Transitions between the different elements were recorded and evaluated with regard to whether they followed a strict cephalocaudal sequence (i.e., no grooming $\rightarrow$ rostral grooming $\rightarrow$ head grooming $\rightarrow$ body grooming $\rightarrow$ no grooming). Any transition that was different from this sequence (i.e., the reversal of such an order or even the skipping of elements; e.g., rostral grooming $\rightarrow$ body grooming) was recorded as "non-cephalocaudal" (i.e., not strictly cephalocaudal). A bout was designated as interrupted if one or more short (up to $5 \mathrm{~s}$ ) pauses occurred between two presentations of the same element or between two different elements. Intervals greater than $5 \mathrm{~s}$ were considered to separate two different bouts. Comparisons were performed with regard to the frequency and duration of the different grooming elements, grooming latency, number of bouts, total duration of grooming, number of transitions between elements, percentage of noncephalocaudal transitions, and percentage of interrupted bouts.

\section{Statistical analysis}

As a preliminary analysis, the number of entries into, time spent on, and distance traveled on the arm to which the animal was confined were compared with the other arm of the same type using $t$-tests for dependent samples. The three groups subjected to different confinement before the elevated plus-maze test were compared either by one-way analysis of variance (ANOVA) or if the data did not meet the normality criteria (evaluated using the Shapiro-Wilk normality test), the Kruskal-Wallis test. Pairwise comparisons were performed using the MannWhitney U test. In addition to these analyses, Spearman's rank correlation coefficients between (1) the percentage of entries into the open arms and (2) the percentage of time on the open arms and grooming measures were calculated with the data from the three groups. In all cases, the significance level was set at $p<0.05$.

\section{Results}

\section{Comparisons between arms of the same type}

No significant differences in exploration (i.e., time spent, entries, and distance traveled) were found between the two open arms in the group previously confined to an open arm. Similarly, exploration of the two closed arms was not different in the group previously confined to a closed arm (data not shown). These results indicate that the maze rotation procedure adopted was at least partially successful in preventing or reversing habituation or learned arm avoidance.

\section{Elevated plus-maze: first 5-min period}

Grooming bouts occurred predominantly in the closed arms and central square. One to three rats in each group exhibited one to two bouts of grooming in the open arms. A preference for grooming in the closed/central area was regularly seen among the groups; therefore, spatial distribution of the bouts was not analyzed.

The conventional measures obtained during the first 5-min period of the elevated plus-maze test are shown in Table 1. ANOVA indicated no effect of prior confinement on the percentage of entries into and percentage of time spent on the open arms or entries into the closed arms. Additionally, no effects on the distance traveled on the arms or entries into their extremities were found. No effect on the time spent in the center square of the maze was found (for all of these measures: $F[2,31]<1.53$, $p>.23)$. Time spent in the extremities of the open or closed arm also showed no effects (Kruskal-Wallis test: $H<1.87, p>.39$ ).

Table 1 also shows the grooming measures obtained during the first 5-min period of the elevated plus-maze test. A significant effect was found in regard to the percentage of interrupted bouts (Kruskal-Wallis test: $H=8.85, p<.05)$. Mann-Whitney U test indicated that the group previously confined to an open arm exhibited a significantly higher percentage of interrupted bouts than the other groups. No effect was found on grooming latency $(F[2,31]=.13, p>.05)$ or other grooming measures during the first 5-min period (Kruskal-Wallis test: $H<3.50, p>.17$ ).

Spearman's rank correlation coefficients are shown in Table 2. The percentage of entries into the open arms was positively correlated with the frequency and duration of the body grooming element.

\section{Elevated plus-maze: second 5-min period}

Grooming bouts occurred predominantly in the closed arms and central square. Only one rat in the group previously confined to an open arm performed one bout in the open arms. The preference for grooming in the closed/central area was again regularly seen among the groups; therefore, the spatial distribution of the bouts was not analyzed.

The conventional measures obtained during the second 5-min period of the elevated plus-maze test are shown in Table 3. Significant effects were found in the percentage of entries into and of time spent on the open arms (Kruskal-Wallis test: $H>6.55, p<.05$ ). In both of these measures the Mann-Whitney $U$ test indicated that the group previously confined to an open arm showed significantly lower values than the group previously confined to a closed arm. No significant effect was found on the distance traveled on the open arms or entries into and time spent in their extremities ( $p=\sim .10)$. No effect was found in the time spent in the center square of the apparatus (for all of these measures, Kruskal-Wallis test: $H<5.57, p>.06)$. Additionally, 
Table 1. Plus-maze measures obtained in the first 5-min period of the test

\begin{tabular}{|c|c|c|c|c|c|c|c|c|}
\hline $\begin{array}{l}\text { Plus-maze measures } \\
\text { Spatiotemporal measures }\end{array}$ & \multicolumn{2}{|c|}{ Familiar cage } & \multicolumn{3}{|c|}{ Closed arm } & \multicolumn{3}{|c|}{ Open arm } \\
\hline OA entries $(\%)$ & 43.0 & $\pm \quad 4.8$ & 46.3 & \pm & 4.0 & 40.9 & \pm & 3.0 \\
\hline OA time $(\%)$ & 29.1 & $\pm \quad 6.0$ & 37.5 & \pm & 6.1 & 27.0 & \pm & 4.2 \\
\hline OA distance traveled (m) & 4.5 & .8 & 5.3 & \pm & .9 & 4.9 & \pm & .6 \\
\hline OA extremity entries & 4.6 & $\pm \quad 1.0$ & 5.7 & \pm & 1.0 & 5.3 & \pm & .6 \\
\hline OA extremity time (s) & 27.3 & $\pm \quad 6.6$ & 42.5 & \pm & 9.6 & 32.3 & \pm & 4.9 \\
\hline CA entries & 8.5 & \pm & 8.4 & \pm & 1.0 & 8.7 & \pm & .6 \\
\hline CA distance traveled $(\mathrm{m})$ & 7.5 & $\pm \quad .6$ & 7.1 & \pm & .7 & 7.8 & \pm & .5 \\
\hline CA extremity entries & 8.6 & .7 & 7.9 & \pm & .9 & 8.9 & \pm & .5 \\
\hline CA extremity time (s) & 75.4 & $\pm \quad 11.9$ & 64.3 & \pm & 12.6 & 85.6 & \pm & 14.7 \\
\hline Time in the center (s) & 45.0 & $\pm \quad 5.7$ & 58.6 & \pm & 8.3 & 42.3 & \pm & 7.0 \\
\hline \multicolumn{9}{|l|}{ Grooming measures } \\
\hline Latency & 130.3 & \pm 22.6 & 135.7 & \pm & 24.3 & 113.4 & \pm & 20.7 \\
\hline Number of bouts & 3.3 & $\pm \quad .6$ & 3.3 & \pm & .6 & 2.9 & \pm & .7 \\
\hline Total duration (s) & 34.2 & $\pm \quad 11.2$ & 22.9 & \pm & 9.9 & 30.1 & \pm & 7.6 \\
\hline Number of pattern transitions & 9.9 & $\pm \quad 2.4$ & 9.5 & \pm & 2.1 & 9.9 & \pm & 1.9 \\
\hline Noncephalocaudal transitions (\%) & 45.3 & $\pm \quad 2.3$ & 47.3 & \pm & 3.5 & 44.8 & \pm & 2.2 \\
\hline Interrupted bouts $(\%)$ & 2.1 & $\pm \quad 2.1$ & 4.5 & \pm & 4.5 & 32.1 & \pm & $11.7 * \mathrm{o}$ \\
\hline Rostral grooming frequency & 3.8 & .7 & 3.6 & \pm & .6 & 4.1 & \pm & .9 \\
\hline Rostral grooming duration (s) & 20.3 & $\pm \quad 3.9$ & 12.2 & \pm & 3.4 & 17.5 & \pm & 4.2 \\
\hline Head grooming frequency & 1.7 & .6 & 1.5 & \pm & .8 & 1.7 & \pm & .3 \\
\hline Head grooming duration (s) & 4.4 & $\pm \quad 1.7$ & 4.0 & \pm & 2.4 & 4.2 & \pm & 1.1 \\
\hline Body grooming frequency & 1.2 & .7 & 1.0 & \pm & .5 & 1.2 & \pm & .5 \\
\hline Body grooming duration (s) & 8.2 & $\pm \quad 5.3$ & 5.8 & \pm & 4.0 & 5.2 & \pm & 2.7 \\
\hline
\end{tabular}

Note: Groups of rats were confined to a familiar cage, closed arm, or open arm prior to the test. Results are expressed as mean $\pm \mathrm{SEM}$. OA, open arm; CA, closed arm.

${ }^{*} p<.05$ compared with the group previously confined to a familiar cage (Mann-Whitney $\mathrm{U}$ test); ${ }^{\circ} p<.05$ compared with the group previously confined to a closed arm (Mann-Whitney U test).

Table 2. Correlations between open-arm exploration (percentages of entries into and time spent on the open arms) and grooming measures during the first 5-min period of the test

\begin{tabular}{lcc}
\hline Grooming measures & \% Entries & \% Time \\
\hline Latency & .04 & .16 \\
Number of bouts & .02 & -.12 \\
Total duration (s) & .09 & -.16 \\
Number of pattern transitions & .12 & -.15 \\
Noncephalocaudal transitions (\%) & -.26 & -.18 \\
Interrupted bouts (\%) & .17 & .08 \\
Rostral grooming frequency & .06 & -.15 \\
Rostral grooming duration (s) & .05 & -.18 \\
Head grooming frequency & .10 & -.25 \\
Head grooming duration (s) & .09 & -.23 \\
Body grooming frequency & $.36^{*}$ & .03 \\
Body grooming duration (s) & $.38^{*}$ & .03 \\
\hline
\end{tabular}

${ }^{*} p \leq .05$ (Spearman test). no significant effects were found on the entries into or distance traveled on the closed arms or entries into their extremities $(F[2,31]<.39, p>.68)$.

The time spent grooming during the second 5-min period of the elevated plus-maze test is shown in Figure 1. A significant effect of prior confinement on the total duration of grooming was found (Kruskal-Wallis test: $H=6.04, p=.05)$. The Mann-Whitney $\mathrm{U}$ test indicated that the group previously confined to an open arm showed higher values than the group confined to a familiar cage. Figure 1 also shows the time spent engaged in each of the constituent grooming elements. Prior confinement significantly affected the time engaged in rostral grooming (Kruskal-Wallis test: $H=6.17, p<.05$ ). The group previously confined to an open arm showed higher values than the other two groups (Mann-Whitney U test). A trend in the same direction was found in the time engaged in body grooming, but this failed to reach significance (Kruskal-Wallis test: $H=5.09, p=.08$ ). No significant effects were found in the other grooming measures (Kruskal-Wallis test: $H<4.96, p>.08$; Table 3). 
Table 3. Elevated plus-maze measures obtained in the second 5-min period of the test

\begin{tabular}{|c|c|c|c|c|c|c|c|c|}
\hline $\begin{array}{l}\text { Plus-maze measures } \\
\text { Spatiotemporal measures }\end{array}$ & \multicolumn{2}{|c|}{ Familiar cage } & \multicolumn{3}{|c|}{ Closed arm } & \multicolumn{3}{|c|}{ Open arm } \\
\hline OA entries (\%) & 25 & \pm & 35 & \pm & 8 & 12 & \pm & $4^{\circ}$ \\
\hline OA time (\%) & 15 & $\pm \quad 5$ & 30 & \pm & 9 & 5 & \pm & $2^{\circ}$ \\
\hline OA distance traveled (m) & 3.0 & $\pm \quad 0.9$ & 3.5 & \pm & 0.9 & .8 & \pm & 0.2 \\
\hline OA extremity entries & 1.6 & \pm 0.5 & 2.0 & \pm & 0.5 & .5 & \pm & 0.2 \\
\hline OA extremity time (s) & 16 & $\pm \quad 5$ & 22 & \pm & 8 & 5 & \pm & 2 \\
\hline CA entries & 4.5 & \pm 0.5 & 4.8 & \pm & 0.8 & 4.0 & \pm & 0.7 \\
\hline CA distance traveled (m) & 5.7 & $\pm \quad 0.7$ & 5.6 & \pm & 1.0 & 4.4 & \pm & 0.5 \\
\hline CA extremity entries & 5.0 & \pm 0.7 & 4.6 & \pm & 0.9 & 4.1 & \pm & 0.6 \\
\hline CA extremity time (s) & 86 & \pm 18 & 91 & \pm & 28 & 154 & \pm & 27 \\
\hline Time in the center (s) & 41 & \pm & 75 & \pm & 23 & 23 & \pm & 8 \\
\hline \multicolumn{9}{|l|}{ Grooming measures } \\
\hline Number of bouts & 1.6 & \pm 0.4 & 2.5 & \pm & 2.8 & 2.8 & \pm & 0.7 \\
\hline Number of pattern transitions & 5.9 & \pm 1.3 & 9.6 & \pm & 13.0 & 13.0 & \pm & 2.8 \\
\hline Noncephalocaudal transitions (\%) & 40.3 & \pm 8.7 & 44.8 & \pm & 46.9 & 46.9 & \pm & 6.6 \\
\hline Interrupted bouts (\%) & 13.9 & \pm 8.7 & 41.2 & \pm & 42.6 & 42.6 & \pm & 12.6 \\
\hline Rostral grooming frequency & 2.1 & \pm 0.5 & 2.7 & \pm & 4.8 & 4.8 & \pm & 1.0 \\
\hline Head grooming frequency & 1.1 & \pm 0.3 & 1.9 & \pm & 2.5 & 2.5 & \pm & 0.7 \\
\hline Body grooming frequency & 1.2 & $\pm \quad 0.4$ & 2.5 & \pm & 2.8 & 2.8 & \pm & 0.7 \\
\hline
\end{tabular}

Note: Groups of rats were confined to a familiar cage, closed arm, or open arm prior to the test. Results are expressed as mean \pm SEM.

OA, open arm; CA, closed arm.

${ }^{\circ} p<.05$ compared with the group previously confined to a closed arm (Mann-Whitney U test).

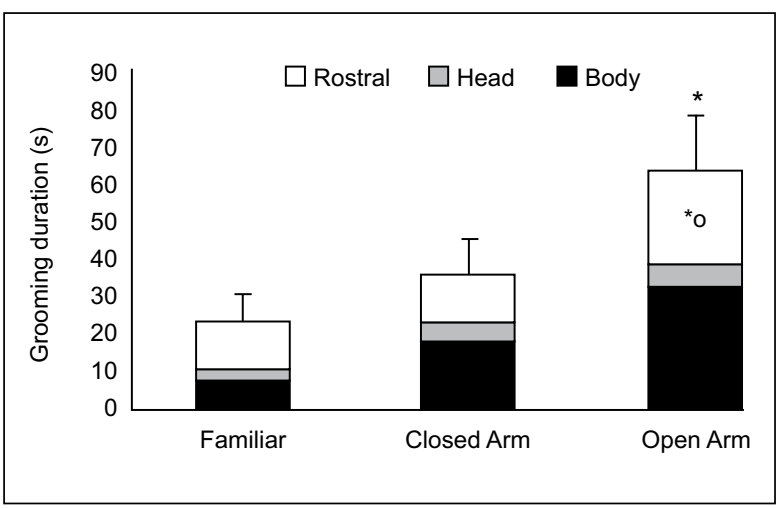

Figure 1. Total time spent grooming and the duration of its constituent elements during the second 5-min period of a $10-$ min elevated plus-maze session. Groups of rats were confined to a familiar cage, closed arm, or open arm prior to the test. $* p<.05$ compared with the group confined to the familiar cage; ${ }^{\bullet} p<.05$ compared with the group confined to the closed arm.

Table 4 shows the Spearman's rank correlation coefficients for the measures obtained during the second 5 -min period. The correlations between the percentage of entries into or percentage of time spent on the open arms and the total duration of grooming barely failed to reach significance $(\rho=-0.322$ and -0.326 , respectively; both $p=.06$ ). If negative associations are found between open-arm exploration and grooming duration, this may be attributable to the time engaged in the rostral and body grooming elements because they showed similar
Table 4. Correlations between open-arm exploration (percentages of entries into and time on the open arms) and grooming measures during the second 5-min period of the test

\begin{tabular}{lcc}
\hline Grooming measures & \% Entries & \% Time \\
\hline Number of bouts & -.08 & .00 \\
Total duration (s) & -.32 & -.32 \\
Number of pattern transitions & -.22 & -.16 \\
Noncephalocaudal transitions (\%) & -.12 & -.01 \\
Interrupted bouts (\%) & .13 & .10 \\
Rostral grooming frequency & -.23 & -.18 \\
Rostral grooming duration (s) & $-.35^{*}$ & $-.34^{*}$ \\
Head grooming frequency & -.22 & -.15 \\
Head grooming duration (s) & -.16 & -.12 \\
Body grooming frequency & -.27 & -.24 \\
Body grooming duration (s) & -.29 & $-.33^{*}$ \\
\hline
\end{tabular}

$* p \leq .05$ (Spearman test).

negative correlations with the percentage of time on the open arms. The time engaged in rostral grooming was also negatively correlated with the percentage of entries into these arms.

\section{Discussion}

The objective of the present study was to investigate the utility of many grooming activity measures in the 
evaluation of anxiety in the elevated plus-maze. Rats were subjected to different confinement procedures known to induce different degrees of aversion. They were subsequently tested in a 10-min elevated plusmaze session that was divided into two blocks. Forced exposure to the comparatively more aversive open arms resulted in some delayed effects (e.g., decreased open arm exploration) that were not detectable in the first 5-min block. This decrease was accompanied by alterations in grooming activity including increased total duration and the duration of rostral grooming. The percentage of interrupted bouts during the first 5-min block was able to detect the effects of prior confinement to an open arm. In addition to these results, correlations were found between open-arm exploration (i.e., the percentages of entries into and time spent on the open arms) and grooming measures, which may be useful in the interpretation of the results.

Although the session was prolonged in an attempt to obtain information about grooming activity in the elevated plus-maze, somewhat surprisingly, this prolongation also revealed information about spatiotemporal measures. This information would not be apparent if the test lasted only $5 \mathrm{~min}$. Confinement to the open arms is a stressful/ anxiogenic experience for rodents. For example, in rats, it leads to anxiety-related behaviors and increases plasma corticosterone concentrations (Pellow et al., 1985). In mice, open-arm confinement increases c-fos expression in neural sites relevant to exploration and anxiety such as the cingulate cortex, nucleus accumbens, dorsal lateral septum, amygdala, and lateral periaqueductal gray (Nguyen et al., 2009).Exposure to an entirely open elevated plus-maze results in antinociception (Cornélio $\&$ Nunes-de-Souza, 2009). The anxious state induced by open-arm confinement in the present work was detectable only in the spatiotemporal measures of openarm exploration during the second half of the 10-min session and not during the first half. This discrepant response to the test between the two parts of the session suggests that anxiety that results from such confinement is a subtle phenomenon that increases with time. The use of a 10-min session for an elevated plus-maze test is quite rare, despite having some advantages (e.g., the apparent nonoccurrence of one-trial tolerance; Holmes $\&$ Rodgers, 1999). In one study in which such a duration was used, the authors chose this test duration "to gain more insight" (p. 54) and in fact were able to show a subtle effect of 3,4-methylenedioxymethamphetamine, which became clear only in the second half of the session (Ludwig, Mihov, \& Schwarting, 2008). Together this previous study and the present study indicate that the duration of exposure to the test may be an important factor in detecting effects in the elevated plus-maze.

The reported effects on open-arm exploration during the second 5-min period were accompanied by marked effects on grooming behavior. The group previously confined to an open arm showed an increased total duration of grooming. This increase was partially attributable to increased rostral grooming. To further explore the link between open-arm exploration and grooming measures, correlation coefficients were calculated. During the first 5-min period, a positive correlation was found between the percentage of entries into the open arms and the body grooming element. During the second 5-min period, the percentage of time on the open arms was negatively correlated with the durations of the rostral and body grooming elements. The duration of rostral grooming was also negatively correlated with the percentage of entries into the open arms. A tentative interpretation of such results with some empirical support is the following.The immediate contact with a challenging context (i.e., the elevated plusmaze) may suppress grooming activity in animals that are more anxious (i.e., the animals that visited the open arms only a few times). In other words, grooming would more likely be seen in the less anxious individuals. The anxious rats would instead dedicate their time to risk assessment. Indeed, rats have been reported to show low levels of grooming when exposed to aversive situations such as a session in a highly illuminated open field (Bouwknecht, Spiga, Staub, Hale, Shekhar, \& Lowry, 2007) or confinement to an open arm (Mairesse et al., 2007). In the present study, although the correlations were consistent with this interpretation, verifying this effect in the group comparison was not possible because the groups did not differ in open-arm exploration during the first 5-min period. Following a period in which risk assessment predominates, the rats that exhibit lower open-arm exploration would be more prone to groom, a behavior that has been implicated in dearousal from stressful situations (Moyaho \& Valencia, 2002; Spruijt et al., 1992). These notions of "grooming dynamics" deserve to be appropriately tested.

Grooming has alternately been endorsed and questioned with regard to its relationship to anxiety. Grooming has been reported to be a behavior that is enhanced by novelty (Jolles, Rompa-Barendregt, \& Gispen, 1979), a response that can be further maximized by prior exposure to a stressful procedure (Katz \& Roth, 1979). The Roman Low and High Avoidance rat lines have been selectively bred for opposite avoidance coping behavior in a two-way active avoidance task, the former line considered to be more anxious. When challenged with novelty, these lines show high and low levels of grooming, respectively (Ferré et al., 1995; Escorihuela et al., 1999). In unselected rats, individual differences in open-arm exploration in the elevated plusmaze are accompanied by grooming behavior with the animals that explore less showing this behavior longer than the other rats (Estanislau et al., 2011). Recently, a "grooming analysis algorithm" was also proposed as a tool in the behavioral evaluation of stress and anxiety (Kalueff \& Tuohimaa, 2004, 2005a). With regard to the questionable validity of grooming measures, the trend toward more ethological approaches to the study of behavior in the elevated plus-maze and other tests that have occurred mainly since the 1990s could have 
revealed a grooming-anxiety relationship. However, the studies did not indicate that grooming behavior is a reliable marker of anxiety. For example, in some factor analyses applied to behavior in the elevated plus-maze, grooming did not load in a factor interpreted as closely related to anxiety (e.g., Cruz et al., 1994; Rodgers \& Johnson, 1995). In an evaluation of serotonin ligands using the elevated plus-maze, none of the five anxiolytic or anxiogenic compounds altered grooming behavior, despite their ability to alter other behaviors (Setem et al., 1999). How can these opposing views of grooming be reconciled? A possible explanation for the confusing or null grooming results that have been reported in studies that used the elevated plus-maze may be the typically short duration of the test session as just discussed above. For grooming behavior the test duration can have great importance. Grooming has long been suggested to be involved in dearousal from stressful situations (for review, see Spruijt et al., 1992). In this regard, a session that is short may only be useful to study arousal-related behavior and not dearousal-related effects that may appear more clearly with a longer session.

Of all the measures taken in the present study, only the proportion of interrupted bouts was able to distinguish prior confinement to an open arm from the other groups within the usual session duration of $5 \mathrm{~min}$ in the elevated plus-maze test. This result appears to replicate the first grooming analysis algorithm studies (Kalueff \& Tuohimaa, 2005a) in which this measure was suggested to be one of the most important in the identification of anxiogenic/anxiolytic effects. The percentage of noncephalocaudal transitions was not affected by prior confinement. This negative result is surprising because such a measure was also proposed to be useful for the study of anxiety (Kalueff \& Tuohimaa, 2005a). In the present study, however, the link between the percentage of noncephalocaudal transitions and anxiety may have been weakened because of differences in the classification of grooming elements from the original studies (Kalueff \& Tuohimaa, 2005a). In the present study, the video recording from above may have impeded the distinction between some elements that were consequently merged together, possibly making the measurement of the proportion of noncephalocaudal transitions less sensitive.

In summary, the present study found that prolongation of the elevated plus-maze session can have heuristic value, allowing the full appearance of subtle effects that are not detected with a shorter session. Grooming activity was found to be sensitive to an anxiogenic experience. Indeed, the percentage of interrupted bouts was useful in the detection of an aversive experience during the first 5-min period of the session. In addition, during the second 5-min period the total duration of grooming and the duration of its rostral element increased after the aversive experience. These results suggest the utility of an in-depth evaluation of grooming behavior in the study of anxiety in the elevated plus-maze and possibly other tests.

\section{Acknowledgements}

We thank Dr. Alberto Fernández-Teruel for helpful comments on an earlier version of the manuscript. The investigation was supported by $\mathrm{CNPq}$ (proc. 481165/2007-0).

\section{References}

Berridge, K. C., Fentress, J. C., \& Parr, H. (1987). Natural syntax rules control action sequence of rats. Behavioural Brain Research, 23, 59-68.

Berridge, K. C., Aldridge, J. W., Houchard, K. R., \& Zhuang, X. (2005). Sequential super-stereotypy of an instinctive fixed action pattern in hyper-dopaminergic mutant mice: A model of obsessive compulsive disorder and Tourette's. BMC Biology, 3, 4.

Bertoglio, L. J., \& Carobrez, A. P. (2000). Previous maze experience required to increase open arms avoidance in rats submitted to the elevated plus-maze model of anxiety. Behavioural Brain Research, 108, 197-203.

Borsini, F., Podhorna, J., \& Marazziti, D. (2002). Do animal models of anxiety predict anxiolytic-like effects of antidepressants? Psychopharmacology, 163, 121-141.

Bouwknecht, J. A., Spiga, F., Staub, D. R., Hale, M. W., Shekhar, A., \& Lowry, C. A. (2007). Differential effects of exposure to low-light or high-light open-field on anxiety-related behaviors: Relationship to c-Fos expression in serotonergic and non-serotonergic neurons in the dorsal raphe nucleus. Brain Research Bulletin, 72, 32-43.

Carobrez, A. P., \& Bertoglio, L. J. (2005). Ethological and temporal analyses of anxiety-like behavior: The elevated plus-maze model 20 years on. Neuroscience and Biobehavioral Reviews, 29, 1193-1205.

Cornélio, A. M., \& Nunes-de-Souza, R. L. (2009). Open elevated plus maze-induced antinociception in rats: A non-opioid type of pain inhibition? Physiology and Behavior, 96, 440-447.

Cruz, A. P. M., Frei, F., \& Graeff, F. G. (1994). Ethopharmacological analysis of rat behavior on the elevated plus-maze. Pharmacology Biochemistry and Behavior, 49, 171-176.

Enginar, N., Hatipoğlu, I., \& Firtina, M. (2008). Evaluation of the acute effects of amitriptyline and fluoxetine on anxiety using grooming analysis algorithm in rats. Pharmacology Biochemistry and Behavior, 89, 450-455.

Escorihuela, R. M., Fernández-Teruel, A., Gil, L., Aguilar, R., Tobeña, A., \& Driscoll, P. (1999). Inbred Roman high- and low-avoidance rats: Differences in anxiety, novelty-seeking, and shuttlebox behaviors. Physiology and Behavior, 67, 19-26.

Estanislau, C., Ramos, A. C., Ferraresi, P. D., Costa, N. F., de Carvalho, H. M. C. P., \& Batistela, S. (2011). Individual differences in the elevated plus-maze and the forced swim test. Behavioural Processes, 86, 46-51.

Ferré, P., Fernández-Teruel, A., Escorihuela, R. M., Driscoll, P., Corda, M. G., Giorgi, O., \& Tobeña, A. (1995). Behavior of the Roman/Verh high- and low-avoidance rat lines in anxiety tests: Relationship with defecation and self-grooming. Physiology and Behavior, 58, 1209-1213.

Jolles, J., Rompa-Barendregt, J., \& Gispen, W. H. (1979). Novelty and grooming behavior in the rat. Behavioral and Neural Biology, $25,563-572$.

Holmes, A., \& Rodgers, R. J. (1999). Influence of spatial and temporal manipulations on the anxiolytic efficacy of chlordiazepoxide in mice previously exposed to the elevated plus-maze. Neuroscience and Biobehavioral Reviews, 23, 971-980.

Kalueff, A. V., \& Tuohimaa, P. (2004). Grooming analysis algorithm for neurobehavioural stress research. Brain Research Protocols, $13,151-158$.

Kalueff, A. V., \& Tuohimaa, P. (2005a).The grooming analysis algorithm discriminates between different levels of anxiety in rats: Potential utility for neurobehavioural stress research. Journal of Neuroscience Methods, 143, 169-177.

Kalueff, A. V., \& Tuohimaa, P. (2005b). Mouse grooming microstructure is a reliable anxiety marker bidirectionally sensitive to GABAergic drugs. European Journal of Pharmacology, 508, 147-153.

Katz, R. J., \& Roth, K. A. (1979). Stress induced grooming in the rat: an endorphin mediated syndrome. Neuroscience Letters, 13, 209-212.

Koolhaas, J. M., de Boer, S. F., Coppens, C. M., \& Buwalda, B. (2010). Neuroendocrinology of coping styles: Towards understanding the 
biology of individual variation. Frontiers in Neuroendocrinology, 31, 307-321.

Ludwig, V., Mihov, Y., \& Schwarting, R. K. W. (2008). Behavioral and neurochemical consequences of multiple MDMA administrations in the rat: role of individual differences in anxiety-related behavior. Behavioural Brain Research,189, 52-64.

Mairesse, J., Viltart, O., Salome, N., Giuliani, A., Catalani, A., Casolini, P., Morley-Fletcher, S., Nicoletti, F., \& Maccari, S. (2007). Prenatal stress alters the negative correlation between neuronal activation in limbic regions and behavioral responses in rats exposed to high and low anxiogenic environments. Psychoneuroendocrinology, 32, $765-776$

Moyaho, A., \& Valencia, J. (2002). Grooming and yawning trace adjustment to unfamiliar environments in laboratory Sprague-Dawley rats (Rattus norvegicus). Journal of Comparative Psychology, 116, 263-269.

Nguyen, N. K., Sartori, S. B., Herzog, H., Tasan, R., Sperk, G., \& Singewald, N. (2009). Effect of neuropeptide Y Y2 receptor deletion on emotional stress-induced neuronal activation in mice. Synapse, 63, 236-246.

Pellow, S., Chopin, P., File, S. E., \& Briley, M. (1985). Validation of open:closed arm entries in an elevated plus-maze as a measure of anxiety in the rat. Journal of Neuroscience Methods, 14, 149-167.

Rodgers, R. J., \& Johnson, J. T. (1995). Factor analysis of spatiotemporal and ethological measures in the murine elevated plus-maze test of anxiety. Pharmacology Biochemistry and Behavior, 52, 297-303.

Setem, J., Pinheiro, A. P., Motta, V.A., Morato, S., \& Cruz, A. P. M. (1999). Ethopharmacological analysis of 5-HT ligands on the rat elevated plus-maze. Pharmacology Biochemistry and Behavior, 62, 515-521.

Spruijt, B. M., van Hooff, J. A. R. A. M., \& Gispen, W. H. (1992). The ethology and neurobiology of grooming behavior. Physiological Reviews, 72, 825-852.

Walf, A. A., \& Frye, C. A. (2007). The use of the elevated plus maze as an assay of anxiety-related behavior in rodents. Nature Protocols, 2, 322-328. 